\title{
Orphan SelD proteins and selenium-dependent molybdenum hydroxylases
}

\author{
Daniel H Haft*1 and William T Self ${ }^{2}$
}

Address: ${ }^{1}$ Department of Bioinformatics, J. Craig Venter Institute, Rockville, MD, 20850, USA and ${ }^{2}$ Department of Molecular Biology and Microbiology, Burnett School of Biomedical Science, College of Medicine, University of Central Florida, Orlando, FL, 32826, USA

Email: Daniel H Haft* - haft@jcvi.org; William T Self - wself@mail.ucf.edu

* Corresponding author

Published: 20 February 2008

Biology Direct 2008, 3:4 doi:10.1186/1745-6150-3-4

This article is available from: http://www.biology-direct.com/content/3/l/4

(c) 2008 Haft and Self; licensee BioMed Central Ltd.

This is an Open Access article distributed under the terms of the Creative Commons Attribution License (http://creativecommons.org/licenses/by/2.0), which permits unrestricted use, distribution, and reproduction in any medium, provided the original work is properly cited.

\begin{abstract}
Bacterial and Archaeal cells use selenium structurally in selenouridine-modified tRNAs, in proteins translated with selenocysteine, and in the selenium-dependent molybdenum hydroxylases (SDMH). The first two uses both require the selenophosphate synthetase gene, selD. Examining over 500 complete prokaryotic genomes finds selD in exactly two species lacking both the selenocysteine and selenouridine systems, Enterococcus faecalis and Haloarcula marismortui. Surrounding these orphan selD genes, forming bidirectional best hits between species, and detectable by Partial Phylogenetic Profiling vs. selD, are several candidate molybdenum hydroxylase subunits and accessory proteins. We propose that certain accessory proteins, and orphan selD itself, are markers through which new selenium-dependent molybdenum hydroxylases can be found.
\end{abstract}

Reviewers: This article was reviewed by Arcady Mushegian and Kira Makarova.

\section{Findings}

A considerable number of selenoproteins are known, in which the sulfur (S) atom of the cysteine side chain is replaced by the analogous but much scarcer element selenium (Se), typically at an enzyme active site. This substitution improves enzyme characteristics [1], such that one in four prokaryotic genomes carries a selenocysteine (SeCys) incorporation system [2,3]. SeCys is incorporated during translation at specific UGA codons as the twentyfirst amino acid in a system that requires a signal from mRNA secondary structure, a special tRNA ( $s e l C)$, the selA (in bacteria), selB, and selD genes [4], and some means of selenium uptake. Species that make even one SeCys-containing protein often make several, with twelve found in Carboxydothermus hydrogenoformans [5]. This pattern suggests that the system may be hard to acquire or maintain, in evolutionary terms, but relatively easy to reuse. SelD makes the activated form of selenium, selenophosphate, and is required also in a fundamentally different system, tRNA modification with selenouridine $[4,6]$. We find the marker specific to that system, 2-selenouridine synthase, in $59 \%$ of genomes with SeCys systems but just $11 \%$ of those without. Such overlapping usage patterns for selenophosphate could mask additional roles for selD.

We searched for orphan (unexplained) selD genes in the first 500 complete prokaryotic genomes and found two instances, one in the bacterium Enterococcus faecalis V583 and one in the archaeon Haloarcula marismortui ATCC 43049. Surrounding the orphan selD genes in both species were proteins related by bi-directional best hits (BBH) between the two genomes. These $\mathrm{BBH}$ pairs included homologs to subunits of a class of molybdenum cofactorcontaining enzymes termed the molybdenum hydroxy- 
lases $(\mathrm{MH})$, plus additional uncharacterized proteins and possible maturations proteins. This finding immediately suggested a biological role for the orphan selD genes, as there are a few known examples of enzymes with a labile selenium atom, which is contributed not by SeCys but through some unknown mechanism. Such enzymes belong to the $\mathrm{MH}$ class, and are designated seleniumdependent molybdenum hydroxylases (SDMH) [7-10]. We noted that various other species carried homologs to several of these BBH hit pairs, typically colocalized with each other, and always in species carrying selD. This observation suggests that the SelD protein, orphan or otherwise, may activate selenium for SDMH maturation. Three SDMH activities have been characterized from two species: nicotinic acid hydroxylase and xanthine dehydrogenase from Eubacterium barkeri $[9,10]$, and xanthine dehydrogenase and purine hydroxylase from Clostridium purinolyticum $[7,8]$. These species also incorporate SeCys; their genome sequences are unavailable, but must carry selD.

The MH contain a catalytically essential, labile (cyanolyzable) sulfur atom, and require shared accessory proteins for enzyme maturation [11]. The labile selenium of the SDMH may have a like need. The few known SDMH act on varied substrates. They may be more similar in sequence to their closest selenium-free homologs than to each other, but likely would share certain selenium insertion proteins. Such accessory proteins, if identified, could serve as genome markers for species producing SeCysindependent selenoenzymes. Several BBH gene pairs in the regions of the two orphan selD genes lack homology to any mature enzyme subunit and are candidate SDMH accessory proteins.

The selD gene is relatively uncommon, occurring in about $30 \%$ of completed prokaryotic genomes. Finding only selD-positive genomes far down a list of BLAST matches would strongly suggest a role in selenium metabolism. Recently, we introduced the method of partial phylogenetic profiling (PPP) [12], which can read this signal. PPP is a means to identify rapidly those protein families closely related in their patterns of distribution to a phenotype, a defined protein family, or a calculated Genome Property $[2,3]$. Phylogenetic profiling techniques, though very powerful in principle [13], rely on good protein families; PPP adjusts the size of each candidate protein family dynamically to find the best match to a profile. For every protein in a query genome, it scores a profile only as large as the working family size (hence "partial") vs. the reference profile, and chooses the size that gives the greatest statistical significance. All genes from a query genome are tested, and ordered according to their individually optimized scores. This method can efficiently rediscover many entire biological systems, such as histidine biosynthesis genes, given a profile based on any one marker gene for the system.

We applied PPP [12] to both the E. faecalis [14] and $H$. marismortui [15] genomes, vs. the profile for selD, using a skew parameter that favors families containing subsets rather than supersets of the species with selD [16]. The top hit in E. faecalis, other than selD itself, was the adjacent gene EF_2566. Though uncharacterized, it shares N-terminal homology (see Pfam model PF01206) [17] with the sulfurtransferase SirA. The selD-dependent species distribution (21 of 21 species) and colocalization with selD in five species strongly suggest that this protein acts in selenium metabolism, though its function remains unknown. Its family is now represented by TIGRFAMs model TIGR03527. The next hit, EF_2568, homologous to cysteine desulfurases and selenocysteine lyases, probably is a selenocysteine lyase. The second hit in H. marismortui, pNG7234, annotated as a phosphate transporter subunit, probably transports selenate as well [18]. These findings show protein families likely involved in selenium metabolism, probably prior to selenophosphate synthesis, and are not pursued further here.

However, the fifth hit in E. faecalis, EF_2563, and the top hit in H. marismortui, pNG7241, are members of the same protein family, TIGR03309. They are found close to selD and form a $\mathrm{BBH}$ pair between the two genomes. The archaeal protein is actually a fusion protein, with its $\mathrm{N}$-terminal half homologous to a xanthine dehydrogenase maturation factor, XdhC [19]. All members of TIGR03309, including YqeB from Escherchia coli, are from species with selD, but then a sharp fall-off in scores occurs before the next set of homologs is encountered, largely from species that lack selD. Reexamination vs. an expanded protein database that includes whole genome shotgun data now confirms that TIGR03309 occurs only in selD-positive genomes. Even after removing redundant sequences with greater than $45 \%$ sequence identity, 26 species remained, leading to vanishingly small odds that TIGR03309 is not closely connected to selenium metabolism. The additional species include a third with an orphan selD gene, Clostridium phytofermentans, a cellulosedegrading, ethanol and hydrogen-producing bacterium [20]; the selD and TIGR03309 genes are one gene apart.

The restriction of the TIGR03309 protein family to selDpositive genomes, frequent fusion with domains homologous to the maturation factor $\mathrm{XdhC}$, and recurring location in extended $\mathrm{MH}$ loci suggest that TIGR03309 members mark a broadly distributed biological system connected to SDMH. To search for components of that system, we applied the PPP algorithm to the profile of TIGR03309 distribution. In each genome tested, most of the top hits fell within a single extended locus. Ten of the 
eleven top hits fall within a 23-gene purine catabolism [21] and selenate reduction [22] region in Escherichia coli. Similarly, the eleven top hits in Aeromonas hydrophila sit together, as do six of the top seven in H. marismortui, seven of nine in Clostridium difficile, etc. These regions typically include two additional uncharacterized families,
TIGR03310 (YgfJ in E. coli) and TIGR03172 (YqeC in E. coli). We propose that these families are also SDMH accessory proteins, possibly for a smaller subset of SDMH than for TIGR03309. Table 1 shows how well the top 25 hits by PPP vs. the TIGR03309 profile mark regions of conserved

Table I: Conserved clustering of top Partial Phylogenetic Profiling results

\begin{tabular}{|c|c|c|c|c|c|c|c|c|c|}
\hline E. coli gene & Putative protein name & PPP rank & E. coli locus & PPP rank & E. faecalis locus & PPP rank & H. maris. locus & PPP rank & C. difficile locus \\
\hline$x d h A$ & $\begin{array}{l}\text { xanthine } \\
\text { dehydrogenase, Mo- } \\
\text { binding subunit }\end{array}$ & 6 & b2866 & 14 & EF_2570 & & & 5 & CD2087 \\
\hline$x d h B$ & $\begin{array}{l}\text { xanthine } \\
\text { dehydrogenase, FAD } \\
\text { binding subunit }\end{array}$ & 15 & b2867 & & & & & & (CD2I0I) \\
\hline $\mathrm{xdhC}$ & $\begin{array}{l}\text { xanthine } \\
\text { dehydrogenase, Fe-S } \\
\text { binding subunit }\end{array}$ & 17 & b2868 & 14 & (EF_2570) & & & & (CD2088) \\
\hline ygeV & $\begin{array}{l}\text { sigma(54)-dependent } \\
\text { activator }\end{array}$ & 13 & b2869 & & & & & & \\
\hline ygeW & carbamoyl transferase & 7 & b2870 & 5 & EF_2577 & & & & \\
\hline ygeX & $\begin{array}{l}\text { diaminopropionate } \\
\text { ammonia-lyase }\end{array}$ & 10 & b287I & 7 & EF_2579 & & & 7 & CD2085 \\
\hline ygeY & $\begin{array}{l}\text { hydrolase, peptidase } \\
\text { M20 family }\end{array}$ & 4 & b2872 & 3 & EF_2578 & & & & (CD2084) \\
\hline ygeZ & phenyl-hydantoinase & 19 & b2873 & & $\left(E F \_2580\right)$ & 6 & pNG7258 & & \\
\hline yqeA & carbamate kinase & 18 & b2874 & 15 & EF_2575 & & & & \\
\hline yqeB & $\begin{array}{l}\text { SDMH accessory } \\
\text { protein TIGR03309 }\end{array}$ & 1 & b2875 & 2 & $E F \_2563$ & $\mathbf{I}$ & pNG724I & $\mathbf{I}$ & CD3478 \\
\hline yqeC & $\begin{array}{l}\text { SDMH accessory } \\
\text { protein TIGR03 I } 72\end{array}$ & 2 & b2876 & 1 & EF_2564 & 2 & pNG7237 & 2 & CD207I \\
\hline ygf] & $\begin{array}{l}\text { SDMH accessory } \\
\text { protein TIGR03310 }\end{array}$ & 5 & b2877 & 21 & EF_2569 & & pNG7236 & & \\
\hline ygfK & $\begin{array}{l}\text { selenate reductase, } \mathrm{Fe}- \\
\mathrm{S} \text { subunit }\end{array}$ & 8 & b2878 & 4 & EF_258I & & & & \\
\hline ygfL & $\begin{array}{l}\text { selenium metabolism } \\
\text { protein SsnA } \\
\text { TIGR033I4 }\end{array}$ & 3 & b2879 & 6 & EF_2582 & 8 & pNG7259 & & \\
\hline ygfM & $\begin{array}{l}\text { selenate reductase, } \\
\text { FAD-binding subunit }\end{array}$ & & b2880 & & & & & & \\
\hline \multirow[t]{3}{*}{ ygfN } & $\begin{array}{l}\text { selenate reductase, } \\
\text { Mo-binding subunit }\end{array}$ & 14 & b288I & & (EF_2570) & 3 & pNG7246 & 3 & (CD2099) \\
\hline & & & & & & 5 & (pNG7244) & 4 & CD2073 \\
\hline & & & & & & & & 5 & (CD2079) \\
\hline \multirow[t]{2}{*}{ yofo } & $\begin{array}{l}\text { xanthine/uracil family } \\
\text { permease }\end{array}$ & & b2882 & & (EF_2573) & & & & \\
\hline & $\begin{array}{l}\text { sulfurtransferase- } \\
\text { related protein } \\
\text { TIGR03527 }\end{array}$ & & & 12 & EF_2566 & & pNG7238 & & CD3667 \\
\hline \multirow[t]{2}{*}{ selD } & $\begin{array}{l}\text { selenophosphate } \\
\text { synthase }\end{array}$ & & bl764 & & EF_2567 & & pNG7239 & & CD2496 \\
\hline & $\begin{array}{l}\text { selenocysteine lyase } \\
\text { TIGR01977 }\end{array}$ & & & 11 & EF_2568 & & & & CD3670 \\
\hline
\end{tabular}

Partial Phylogenetic Profiling results for Escherichia coli, Enterococcus faecalis, Haloarcula marismortui, and Clostridium difficile vs. the profile of the putative SDMH accessory protein family TIGR03309. The first seventeen rows show a contiguous gene region in E. coli, and the last three a region in $E$. faecalis. The first two columns show $E$. coli gene symbols and putative protein names. The third, fifth, seventh, and ninth columns represent rank by PPP for $E$. coli (out of 4243 proteins), E. faecalis (3277 proteins), H. marismortui ( 4240 proteins - all loci found are on plasmid with 362 proteins), and C. difficile (3873 proteins); missing values show that the protein did not score in the top twenty-five. Locus tags shown in columns 6,8 , and 10 mark genes with bi-directional best hits vs. the corresponding $E$. coli locus in column 4 and co-clustered in their respective species, so not all of the top 25 hits appear in the table; locus tags in parentheses are unidirectional best hits from the E. coli gene. The row for putative SDMH accessory protein family TIGR03309 is in boldface. The putative $C$. difficile ortholog to the $C$. purinolyticum xanthine dehydrogenase molybdenum-binding subunit is in italicized boldface. 
gene clustering between E. coli,E. faecalis, H. marismortui, and C. difficile.

TIGR03309 clearly is connected both to selenophosphate synthase activity and to molybdenum hydroxylases. In theory, the connection might be that TIGR03309 represents an accessory protein to an apparent selenate reductase found nearby in E. coli, in a selenium detoxification system that requires activation by SelD. Searching for homologs to selenate reductase subunits, however, pulls in few genomes with both TIGR03309 and SelD before hitting large numbers of genomes with neither. Therefore it seems more likely that the selenate reductase helps furnish selenide to SelD in select species, while TIGR03309 family members contribute to SDMH maturation. While no complete genome sequence is available for either genome with characterized SDMH, N-terminal sequences are available for $C$. purinolyticum selenium-dependent xanthine dehydrogenase $(\mathrm{XDH})$ subunits, from which we identify putative orthologs in the nearly complete genome of $C$. difficile. The top hit there, after the TIGR03309 and TIGR03172 family putative SDMH accessory proteins, is the apparent ortholog to the characterized XDH molybdenum-binding subunit. This last observation appears to cement the connection of SDMH with both TIGR03309 and selD, and suggests a means to discover new seleniumdependent molybdenum hydroxylases. This class of enzyme may be more common than previously appreciated, and may involve purine catabolic systems in gut bacteria such as E. coli. This notion is intriguing because a defect in purine metabolism causes gout; human health therefore may be affected by selenium availability to gut bacteria.

\section{Competing interests}

The author(s) declare that they have no competing interests.

\section{Authors' contributions}

$\mathrm{DH}$ performed the analyses. $\mathrm{DH}$ and WS interpreted the results. DH wrote the paper. DH and WS edited the paper.

\section{Reviewers' comments Reviewer I: Arcady Mushegian, Stowers Institute for Medical Research}

The note reports several observations that are of interest. I recommend the following modifications.

1. As it is currently written, the report is mostly chronological: it starts with the observations were made first, follows with other observations they led to, etc. I suppose that the notes on computational prediction may benefit from following a different plan, which is a bit more formal: first state all predictions that you are making, then show what is the evidence supporting these predictions, and then tell why alternative explanations of the same evidence are less likely than the initial hypothesis. This will make the logic much easier to follow.

2. This work: Lobanov AV, Hatfield DL, Gladyshev VN. Selenoproteinless animals: Selenophosphate synthetase SPS1 functions in a pathway unrelated to selenocysteine biosynthesis. Protein Sci. 2008 Jan;17(1):176-82. appears to be directly relevant. Please consider discussing it.

3. Ygf is quite clearly a nucleotidyltransferase of dinucleotide sugar transferase Rossmanoid fold (verify by PSIBLAST or HHPred). This can be worked into the pathway reconstruction.

\section{Authors' response}

The highly compressed format of the Discovery Note departs somewhat from the style of longer papers. We have clarified the discussion of the most likely alternative hypothesis, namely that TIGR03309 members are accessory proteins for molybdenum hydroxylases that detoxify selenate in a process that further requires activation by SelD, rather than accessory proteins for molybdenum hydroxylases that contain selenium. This alternative hypothesis does not seem strong because the molybdopterin-containing enzymes that accompany TIGR03309 are varied and do not lead to the identification of protein families exclusively in SelD-positive genomes; only our putative accessory protein families, TIGR03309 in particular, do that. On the other hand, our proposed role for TIGR03309 in SDMH maturation suggests a labile selenium-dependent enzyme in $E$. coli, something not yet observed, so alternative hypotheses must be considered.

The note on animals with selenophosphate synthetase but no selenocysteine is consistent with several scenarios, the most likely being a role in the selenouridine modification to tRNA, which does occur in animals. A seleniumdependent molybdenum hydroxylase system is possible, but TIGR03309 and other putative SDMH markers hit no animal proteins.

The molybdenum-containing cofactors in seleniumdependent and other molybdenum hydroxylases are molybopterin derivatives, complex in their structure and variable between enzymes. In the SDMH nicotinic acid hydroxylase from E. barkeri, for example, Gladyshev and Lecchi identified molybdopterin cytosine dinucleotide. A nucleotidyltransferase role is therefore fully consistent with a role for YgfJ and other members of TIGR03310 as SDMH accessory proteins. 


\section{Reviewer 2: Kira S. Makarova, National Center for Biotechnology Information}

In this manuscript several considerations are described in support of the hypothesis that selenophosphate synthase SelD is not only the necessary component of selenouridine and selenocysteine systems but is also involved in formation of (or activation the selenium for) molybdenum hydroxylases, some of which are shown experimentally to be selenium-dependent.

The observation that two (out of $\sim 500$ analyzed) organisms with completely sequenced genomes contain the selD-like gene but neither genes for selenouridine and selenocysteine systems prompted the authors to investigate the neighborhood of these "orphan" selD in these two genomes in order to find a system where the SelD protein may function in these organisms. In the close vicinity of selD gene the homologs of the selenium dependent molybdenum hydroxylases (SDMH) can be found in both genomes suggesting a possible link between SelD and SDMH in these two genomes. This led the authors to hypothesis that these enzymes are likely seleniumdependent and SelD might be involved in the selenium activation for these enzymes.

One way to further support this hypothesis is to consider the inferred operon organization of the SelD-SDMH genome neighborhood. If selD could be predicted to cotranscribe with SDMH genes it would lend additional weight to the author's arguments. However these genes are co-directed and separated by a short ( $<100$ bp) intergenic spacers only in E. faecalis while the neighborhood pattern in $H$. marismortui is incompatible with co-transcription and/or co-regulation. This lack of conservation of the selD-SDMH neighborhood weakens the evidence of their functional connection. It will be interesting to check all other genomes containing both selD and SDMH genes if there are any other cases where these genes might be inferred as co-transcribed or co-regulated.

Co-occurrence is another way to predict a functional link between genes, but it is particularly hard to use this evidence for functional linking of protein families, especially for those with large number of paralogs. The authors admit that the Phylogenetic Profiling methods rely on "good" protein families, but do not provide any considerations if the families or subfamilies investigated here are "good" or "bad". In this respect it is better not to go into details describing the advantages or drawbacks of the particular method but rather make a safe statement that PPP results are used essentially for enriching the set by the genes with possible functional link for further analysis. The parameters of the PPP search which can be used for reproducing the reported results are however of interest.
It should be explicitly stated why the authors do not show all the top 25 results of Partial Phylogenetic Profiling but only some selected subset. Apparently they do not believe that the omitted genes are relevant to selenium metabolism. Ideally, both the original data and the author's rationale for including or discarding a gene should be available to readers.

The strongest evidence that is presented in the paper is the notion that TIGR03309 is found only in the genomes containing SelD. Since the genomes that encode for TIGR03309 family proteins belong to a variety of bacterial clades there is a little doubt that the connection between SelD and TIGR03309 family is real and strong. But is this connection direct or indirect? If most of these organisms share an ecological niche or lifestyle it is possible that they would share a number of functionally unrelated genes due to shared physiologic requirements or simply physical proximity.

The authors explain the observed connection between SelD and TIGR03309 family by hypothesizing that TIGR03309 proteins recruit selenophosphate for use in $\mathrm{SDMH}$ system. Are there other reasonable alternative hypotheses? How do the authors discriminate between them?

I believe however that despite the relatively weak support, the hypothesis described here is interesting, entirely reasonable and is an excellent starting point for further investigation by both experimental and computational means.

\section{Authors' response}

Dr. Makarova is correct to point out that the circumstantial evidence supporting TIGR03309 as a marker for selenium dependent molybdenum hydroxylases, though substantial, is not yet sufficient to eliminate doubt. We formed our hypotheses based on the number of genomes we could investigate with PPP, a tool that exploits precomputed all-vs.-all BLAST comparison results. We waited, however, until more whole genome shotgun sequences became available so we could test TIGR03309 against SelD to a much greater depth in non-redundant sequences. These added genomes include Clostridium phytofermentans ISDg, the third instance of an orphan SelD genome, taxonomically quite different from the other two. In genomes with either selenocysteine or selenouridine systems, selD sits far from the TIGR03309 family gene, but in C. phytofermentans, the three occur in the gene order selD - TIGR03527 - TIGR03309, and once again near xanthine dehydrogenase homologs. This is the third different arrangement for selD and TIGR03309 in three orphan SelD genomes (one gene between, three genes between, and adjacent but converging), but they are always close. We are not troubled by the lack of conserved operon struc- 
ture because there is no reason to expect similar expression levels of SelD and TIGR03309 to be required. We have somewhat expanded the discussion of C. phytofermentans.

The taxonomies of TIGR03309-containing species are quite diverse, but so are their environmental niches. Several represent human intestinal bacteria, while others include a Dead Sea halophilic archaeon, a carbon monoxide-metabolizing thermophile, a ubiquitous water-borne mesophile, a metal-reducing delta proteobacterium, etc. We think it unlikely the link to SelD represents an indirect link to a common environmental factor.

We agree with Dr. Makarova's assertion (except for top hits with stand-out statistical significance) that the PPP algorithm does not guarantee a meaningful link between a protein family and a biological process, but rather enriches for proteins likely to be relevant. PPP results may reflect both false-positives and false-negatives for proteins that are not simultaneously required for and restricted to the occurrence of a system in a genome. Table 1 therefore cleans the signal slightly by imposing two requirements. Proteins are shown if they are identifiable as parts of conserved gene regions shared with E. coli (top 17 rows) and E. faecalis (bottom 3 rows), and their PPP rank is shown if in the top 25. We have tried to make the legend for table 1 clearer.

\section{Acknowledgements}

We would like to thank Jeremy Selengut for detailed discussions and for extensions to the partial phylogenetic profiling program. This work was supported by grants from the United States National Science Foundation (DBI-0445826) and the United States Department of Energy (DE-FG0204ER63935).

\section{References}

I. Wessjohann LA, Schneider A, Abbas M, Brandt W: Selenium in chemistry and biochemistry in comparison to sulfur. Biol Chem 2007, 388(10):997-1006.

2. Haft DH, Selengut JD, Brinkac LM, Zafar N, White O: Genome Properties: a system for the investigation of prokaryotic genetic content for microbiology, genome annotation and comparative genomics. Bioinformatics 2005, 2 I (3):293-306.

3. Selengut JD, Haft DH, Davidsen T, Ganapathy A, Gwinn-Giglio M, Nelson WC, Richter AR, White O: TIGRFAMs and Genome Properties: tools for the assignment of molecular function and biological process in prokaryotic genomes. Nucleic Acids Res 2007, 35(Database issue):D260-4.

4. Romero H, Zhang Y, Gladyshev VN, Salinas G: Evolution of selenium utilization traits. Genome Biol 2005, 6(8):R66.

5. Wu M, Ren Q, Durkin AS, Daugherty SC, Brinkac LM, Dodson RJ, Madupu R, Sullivan SA, Kolonay JF, Haft DH, Nelson WC, Tallon LJ, Jones KM, Ulrich LE, Gonzalez JM, Zhulin IB, Robb FT, Eisen JA: Life in hot carbon monoxide: the complete genome sequence of Carboxydothermus hydrogenoformans Z-290I. PLoS Genet 2005, I(5):e65.

6. Veres Z, Stadtman TC: A purified selenophosphate-dependent enzyme from Salmonella typhimurium catalyzes the replacement of sulfur in 2-thiouridine residues in tRNAs with selenium. Proc Natl Acad Sci U S A 1994, 9 I ( I 7):8092-8096.

7. Self WT, Stadtman TC: Selenium-dependent metabolism of purines: A selenium-dependent purine hydroxylase and xan- thine dehydrogenase were purified from Clostridium purinolyticum and characterized. Proc Natl Acad Sci U S A 2000, 97(I3):7208-72। 3.

8. Self WT, Wolfe MD, Stadtman TC: Cofactor determination and spectroscopic characterization of the selenium-dependent purine hydroxylase from Clostridium purinolyticum. Biochemistry 2003, 42(38): I I 382- I I 390.

9. Gladyshev VN, Khangulov SV, Stadtman TC: Nicotinic acid hydroxylase from Clostridium barkeri: electron paramagnetic resonance studies show that selenium is coordinated with molybdenum in the catalytically active seleniumdependent enzyme. Proc Natl Acad Sci U S A 1994, 9 I ( I):232-236.

10. Schrader T, Rienhofer A, Andreesen JR: Selenium-containing xanthine dehydrogenase from Eubacterium barkeri. Eur J Biochem 1999, 264(3):862-87|.

II. Amrani L, Primus J, Glatigny A, Arcangeli L, Scazzocchio C, Finnerty $\checkmark$ : Comparison of the sequences of the Aspergillus nidulans hxB and Drosophila melanogaster ma-l genes with nifS from Azotobacter vinelandii suggests a mechanism for the insertion of the terminal sulphur atom in the molybdopterin cofactor. Mol Microbiol 2000, 38(I): I| |4- I25.

12. Haft DH, Paulsen IT, Ward N, Selengut JD: Exopolysaccharideassociated protein sorting in environmental organisms: the PEP-CTERM/EpsH system. Application of a novel phylogenetic profiling heuristic. BMC Biol 2006, 4:29.

13. Pellegrini M, Marcotte EM, Thompson MJ, Eisenberg D, Yeates TO: Assigning protein functions by comparative genome analysis: protein phylogenetic profiles. Proc Natl Acad Sci U S A 1999 , 96(8):4285-4288.

14. Paulsen IT, Banerjei L, Myers GS, Nelson KE, Seshadri R, Read TD, Fouts DE, Eisen JA, Gill SR, Heidelberg JF, Tettelin H, Dodson RJ, Umayam L, Brinkac L, Beanan M, Daugherty S, DeBoy RT, Durkin S, Kolonay J, Madupu R, Nelson W, Vamathevan J, Tran B, Upton J, Hansen T, Shetty J, Khouri H, Utterback T, Radune D, Ketchum KA, Dougherty BA, Fraser CM: Role of mobile DNA in the evolution of vancomycin-resistant Enterococcus faecalis. Science 2003, 299(56 I 5):207|-2074.

15. Baliga NS, Bonneau R, Facciotti MT, Pan M, Glusman G, Deutsch EW, Shannon P, Chiu Y, Weng RS, Gan RR, Hung P, Date SV, Marcotte E, Hood L, Ng WV: Genome sequence of Haloarcula marismortui: a halophilic archaeon from the Dead Sea. Genome Res 2004, I 4(I I):222I-2234.

16. Selengut JD: unpublished. 2007.

17. Finn RD, Tate J, Mistry J, Coggill PC, Sammut SJ, Hotz HR, Ceric G, Forslund K, Eddy SR, Sonnhammer EL, Bateman A: The Pfam protein families database. Nucleic Acids Res 2008, 36(Database Issue):D28I-D288. Epub 2007 Nov 26.

18. Li HF, McGrath SP, Zhao FJ: Selenium uptake, translocation and speciation in wheat supplied with selenate or selenite. New Phytol 2008.

19. Neumann $M$, Schulte $M$, Junemann $N$, Stocklein $W$, Leimkuhler S: Rhodobacter capsulatus $X \mathrm{dhC}$ is involved in molybdenum cofactor binding and insertion into xanthine dehydrogenase. J Biol Chem 2006, 28 I(23): I 570 I- I 5708.

20. Warnick TA, Methe BA, Leschine SB: Clostridium phytofermentans sp. nov., a cellulolytic mesophile from forest soil. Int J Syst Evol Microbiol 2002, 52(Pt 4): I I 55- I I 60.

21. Xi H, Schneider BL, Reitzer L: Purine catabolism in Escherichia coli and function of xanthine dehydrogenase in purine salvage. J Bacteriol 2000, I 82( I 9):5332-534I.

22. Bebien M, Kirsch J, Mejean V, Vermeglio A: Involvement of a putative molybdenum enzyme in the reduction of selenate by Escherichia coli. Microbiology 2002, I 48(Pt I 2):3865-3872. 